\title{
Costo institucional del paciente con incapacidad temporal para el trabajo por lumbalgia mecánica
}

\author{
MARÍA DEL CARMEN PONCE MARTÍNEZ,;*** ENRIQUE VILLARREAL-RÍOS* \\ EMMA ROSA VARGAS-DAZA, ${ }^{*}$ LIDIA MARTÍNEZ-GONZÁLEZ* y LILIANA GALICIA-RODRÍGUEZ* \\ "Unidad de Investigación Epidemiológica y en Servicios de Salud Querétaro \\ del Instituto Mexicano del Seguro Social, México \\ **Unidad de Medicina Familiar No. 16 Querétaro, Instituto Mexicano del Seguro Social
}

\begin{abstract}
RESUMEN
Introducción: La lumbalgia afecta de manera frecuente a la población económicamente activa. Es la primera causa de pérdida de días laborales en trabajadores menores de 55 años de edad y la segunda de ausentismo laboral.

Objetivo: Determinar el costo institucional del paciente con incapacidad temporal para el trabajo por lumbalgia mecánica.

Materiales y métodos: Se realizó un estudio de costos de expedientes de trabajadores con lumbalgia de 20 a 60 años con incapacidad temporal para el trabajo. El tamaño de la muestra (228 pacientes) se calculó con la fórmula de promedios para población infinita con nivel de confianza del $95 \%$. La técnica muestral fue por cuota empleando como marco muestral el listado de pacientes con incapacidad temporal para el trabajo y diagnóstico de lumbalgia mecánica. Las variables estudiadas fueron las características sociodemográficas, el perfil de uso, costo unitario y costo promedio de los servicios otorgados. El análisis estadístico incluyó porcentajes, promedios, intervalo de confianza y proyección.
\end{abstract}

Resultados: El costo total promedio por paciente con lumbalgia mecánica es de 1744,08 USD, la incapacidad es el costo más elevado (1083,71 USD), seguido de los estudios de gabinete (394,89 USD), las consultas (180,52 USD), los medicamentos y el laboratorio.

Conclusión: El costo institucional del paciente con incapacidad temporal para el trabajo con lumbalgia mecánica

Recibido el 5-3-2013. Aceptado luego de la evaluación el 30-5-2013. Correspondencia: Dr. ENRIQUE VILLARREAL-RÍOS felibree@infosel.net.mx resulta en miles de dólares y, proyectado a una población de pacientes, el costo se incrementa a millones.

\section{Palabras Clave: Lumbalgia. Costo. Incapacidad.}

INSTITUTIONAL COST OF THE PATIENT WITH TEMPORARY INABILITY TO WORK DUE TO MECHANICAL LOW BACK PAIN

ABSTRACT
Background: Back pain affects frequently to the econo-
mically active people. It is the first cause of business day
loss in workers aged 55 or less, and the second cause of
absenteeism. Objective: To determine the institutional cost of the patient with temporary inability to work, due to low back pain.

Methods: Costs design records of patients $<55$ years old, with temporary inability to work were analyzed. The sample size (228 patients) was calculated with the average formula for infinite people, with confidence interval of $95 \%$. The sample technique was by quota, using the listing of patients with temporary inability to work and mechanical low back pain, as framework. Variables used were socio-demographic characteristics, unit cost and average cost of services provided. Statistical analysis included percentages, means, confidence interval and projection.

Results: Total average cost of the patient with mechanical low back pain is about 1,744.08 USD, the inability represents the highest average cost (1,083.71 USD), followed by cabinet studies (394.89 USD), appointments (180.52 USD), medications and laboratory.

Conclusion: The institutional cost of the patient with inability to work secondary to low back pain is high and the cost increases to millions in a population of patients. 
KEY WORDS: Low back pain. Costs. Inability.

\section{Introducción}

La lumbalgia es un problema de salud pública que afecta, de manera frecuente, a la población económicamente activa, es decir, a un cuarto de la población general. Se identifica como la primera causa de pérdida de días laborales en trabajadores menores de 55 años de edad, y la segunda causa de ausentismo laboral, sólo después del resfriado común. Actualmente se considera que, cada año, cerca del $50 \%$ de las personas laboralmente activas sufre un episodio de lumbalgia..$^{1-3}$

Alrededor del $90 \%$ de las lumbalgias se deben a una alteración mecánica, el 10\% restante corresponde a patología no mecánica o bien a patología ajena a la columna. El dolor lumbar crónico puede provocar discapacidad, consecuencias físicas y psicológicas para el paciente, además de generar importantes desafíos económicos. ${ }^{4}$

Cuando un trabajador se encuentra incapacitado para trabajar de manera temporal, es necesario justificar su inasistencia por medio de incapacidad durante los días que requiera para su recuperación. Los tipos de incapacidad son la permanente parcial (IPP) y temporal para el trabajo (ITT), esta última, a su vez, se clasifica según si es por enfermedad general correspondiente a un $60 \%$ del salario a partir del cuarto día; en cambio, si es por riesgo de trabajo es el $100 \%$ del salario cotizado por el patrón de la empresa. La ITT es una prestación de seguridad social que cubre el riesgo de pérdida de ingresos por problemas de salud, causados por una enfermedad o un accidente, laboral o no laboral. Además de los días de incapacidad, el manejo de la lumbalgia crónica conlleva gastos de medicamentos, gabinete, laboratorio y consultas. ${ }^{5,6}$

Se desconoce el costo de un paciente con lumbalgia mecanopostural a lo largo de su evolución y, por consiguiente, la magnitud monetaria que conlleva cuando el paciente se encuentra con incapacidad prolongada. Por lo tanto, el objetivo de este trabajo fue determinar el costo institucional del paciente con incapacidad temporal para el trabajo debido a lumbalgia mecánica.

\section{Métodos}

Se realizó un estudio de costo de la atención del paciente con lumbalgia mecánica durante el período comprendido entre enero de 2010 y agosto de 2012, en el Instituto Mexicano del Seguro Social, en la ciudad de Querétaro.

Se incluyeron expedientes de pacientes de 20 a 60 años, de ambos sexos, trabajadores con cotización directa a la institución de salud, a quienes se les hubiera otorgado ITT remunerada. Se eliminaron los expedientes con datos incompletos.
El tamaño de la muestra (228 pacientes) se calculó con la fórmula de promedios para población infinita con intervalo de confianza del $95 \%$ para una cola $\mathrm{Z}=1,64$; costo promedio institucional 5000 USD, desviación estándar 2968 y margen de error de 323.

La técnica muestral fue por cuota empleando como marco de la muestra el listado de pacientes con ITT y diagnóstico de lumbalgia mecánica.

Las variables estudiadas incluyeron características sociodemográficas: edad, sexo, estado civil, escolaridad y ocupación.

Costo promedio. El costo promedio se estimó a partir del perfil de uso y el costo unitario del servicio de consulta externa de Medicina Familiar, Traumatología, Medicina Física, Laboratorios, Estudio de Gabinete, medicamentos prescritos y días de incapacidad.

Perfil de uso. Para identificar el perfil de uso se consultó la base de datos del Servicio de Medicina del Trabajo identificando todos los expedientes con diagnóstico de lumbalgia; aunado a ello, se contabilizó el número de días de incapacidad. Posteriormente se identificaron la cantidad de consultas recibidas, los estudios de gabinete, laboratorio y los medicamentos en el expediente de Medicina Familiar. Cuando el paciente se refirió a los servicios de Traumatología y Medicina Física se contabilizó el número de atenciones recibidas hasta su alta.

Costo unitario. El costo unitario de los servicios de Medicina Familiar, Medicina Física, Traumatología, Laboratorio se estimó con la técnica de tiempos y movimientos a partir del ingreso mensual del personal de salud y los recursos físicos, considerando el tiempo laborado y el costo por minuto. Luego, se identificó la cantidad de minutos dedicados al paciente en cada uno de los Servicios ya mencionados. El costo de los estudios de gabinete y medicamentos se obtuvo del listado nominal existente del departamento de administración. El costo del día de incapacidad se adquirió del programa de incapacidades de Medicina del Trabajo.

A fin de estimar el costo promedio de la ITT se clasificó al grupo de estudio en pacientes con lumbalgia por riesgo de trabajo, enfermedad general y riesgo de trabajo con enfermedad general. Para riesgo de trabajo se consideró a aquellos pacientes que la presentan en el momento de las actividades laborales o bien durante el trayecto de su casa al trabajo y del trabajo a su casa; en este tipo de paciente, la incapacidad se paga al $100 \%$ del salario cotizado. El segundo grupo se clasificó a partir de causas no relacionadas a actividades laborales y cubriendo el $60 \%$ del salario cotizado a partir del cuarto día. El tercer grupo se integró con ambos tipos de incapacidad, debido al tiempo de espera antes de calificarla como riesgo de trabajo se cotiza el $60 \%$ del salario a partir del cuarto día y después el 100\% cuando se califica como riesgo de trabajo.

Finalmente se concentró la información de los expedientes en una base de datos y se analizó. El análisis esta- 
dístico incluyó porcentajes, promedios, intervalo de confianza, proyección.

\section{Resultados}

Se estudiaron 228 pacientes con lumbalgia mecánica e ITT. El promedio de edad era de 38.09 años (IC 95\% 31.8-44.4), con predominio del sexo masculino 58,3\% (IC 95\% 51,9-64,7), casados 55,4\% (IC 95\% 48,9-61,9) y escolaridad secundaria $31,9 \%$ (IC 95\% 25,8-38,0).

La lumbalgia posesfuerzo fue la más frecuente, seguida de la lumbociática y la hernia discal. En la Tabla 1, se muestra la prevalencia por tipo de lumbalgia.

La consulta más solicitada correspondió a Medicina Familiar, con un costo total por paciente de 85,84 USD, le seguían Traumatología y Ortopedia y, por último, Medicina Física y Rehabilitación. Del total de estudios solicitados, la radiografía simple fue la más requerida por el médico, con un costo unitario de 62,64 USD y un costo total de 143,46 USD; en tanto, la electromiografía tuvo un menor uso, costo unitario 37,71 USD y costo total 38,84 USD. El medicamento que implica un mayor costo por su prescripción fue la gabapentina. En la Tabla 2, se presenta el uso, el costo unitario y el costo total por uso de servicios otorgados.

El número de días y el costo total es mayor en los pacientes con incapacidad por riesgo de trabajo: el 13,15\% de los pacientes con este tipo de incapacidad redunda en un costo de 1563,03 USD. El 84,21\% tenía incapacidad por enfermedad general, con un costo de 1019,99 USD por paciente; existe un tercer grupo que integra incapacidad por enfermedad general y riesgo de trabajo, con un costo de 731,57 USD, formado por el 2,63\% de los pacientes. El costo promedio total es 1083,71 USD. En la Tabla 3, se detallan los días y el costo unitario por tipo de incapacidad.

El costo total de un paciente con lumbalgia mecánica es de 1722,9 USD, la incapacidad genera el costo más elevado 1083,70 USD, seguida de los estudios de gabinete 394,89 USD, las consultas 180,52 USD, los medicamentos y el laboratorio. En la Tabla 4, se muestra el costo total del paciente con lumbalgia mecánica.
La proyección a 2000 pacientes representa un costo total de 3.445.794,71 USD; la incapacidad, un costo promedio de \$2.167.400,00 USD, seguida de estudios de radiografía y consulta de Medicina Familiar. En la Tabla 5, se presenta la proyección del costo promedio por servicio de 2000 pacientes.

\section{Discusión}

La lumbalgia es considerada un problema de salud en el mundo, pues afecta a un cuarto de la población general, además de ser un padecimiento antiguo y frecuente de la humanidad. Se identifica como la primera causa de pérdida de días laborales en trabajadores menores de 55 años de edad, y la segunda causa de ausentismo laboral. ${ }^{7}$

En las sociedades occidentales, la incidencia varía entre el $60 \%$ y el $90 \%$, los datos advierten que entre el $55 \%$ y el $80 \%$ de las personas se verán incapacitadas, al menos, una vez en la vida. ${ }^{3}$

La importancia del análisis de costos en pacientes con lumbalgia e incapacidad laboral dentro del sistema de salud tiene relevancia, debido a los escasos estudios sobre el tema, su impacto en el paciente y en los recursos institucionales por incapacidad.

La prevalencia de lumbalgia durante toda la vida es del $60 \%-80 \%$ y la tasa de incidencia, del 5\%-25\% entre los 25-45 años, sin predominio de sexo. En 2002, Saldivar comunicó que la prevalencia es más alta en el sexo masculino, y que aparece entre los 30 y 39 años de edad; en el presente estudio, se hallaron datos similares, con un promedio de edad de 38 años, y predominio del sexo masculino, debido a las actividades laborales desempeñadas. Además, cabe señalar que el promedio de edad ubica a esta población en la categoría de adultos jóvenes, productivos, lo que puede significar que a menor edad se incapaciten, por lo cual tienen más probabilidad de incapacitarse a lo largo de su vida laboral, generando costos a la institución responsable de su cuidado. Se estima que al trabajador con lumbalgia se le llegan a otorgar hasta 102 días en promedio, lo que resulta en un costo de 252,95 USD por episodio agudo, dato que coincide con los días reportados en el presente estudio. ${ }^{1,8,9}$

Tabla 1. Prevalencia por tipo de lumbalgia

\begin{tabular}{|c|c|c|c|}
\hline \multirow{2}{*}{ Tipo de lumbalgia } & \multirow{2}{*}{ Porcentaje } & \multicolumn{2}{|c|}{ IC $95 \%$} \\
\hline & & Inferior & Superior \\
\hline Lumbalgia posesfuerzo & 59,2 & 52,8 & 65,6 \\
\hline Hernia discal & 18,4 & 13,4 & 23,4 \\
\hline Canal lumbar estrecho & 2,2 & 0,3 & 4,1 \\
\hline Lumbociática & 18,9 & 13,8 & 24,0 \\
\hline Artrosis interapofisaria & 1,3 & 0,2 & 2,8 \\
\hline
\end{tabular}


Tabla 2. Costo total por uso de servicios otorgados

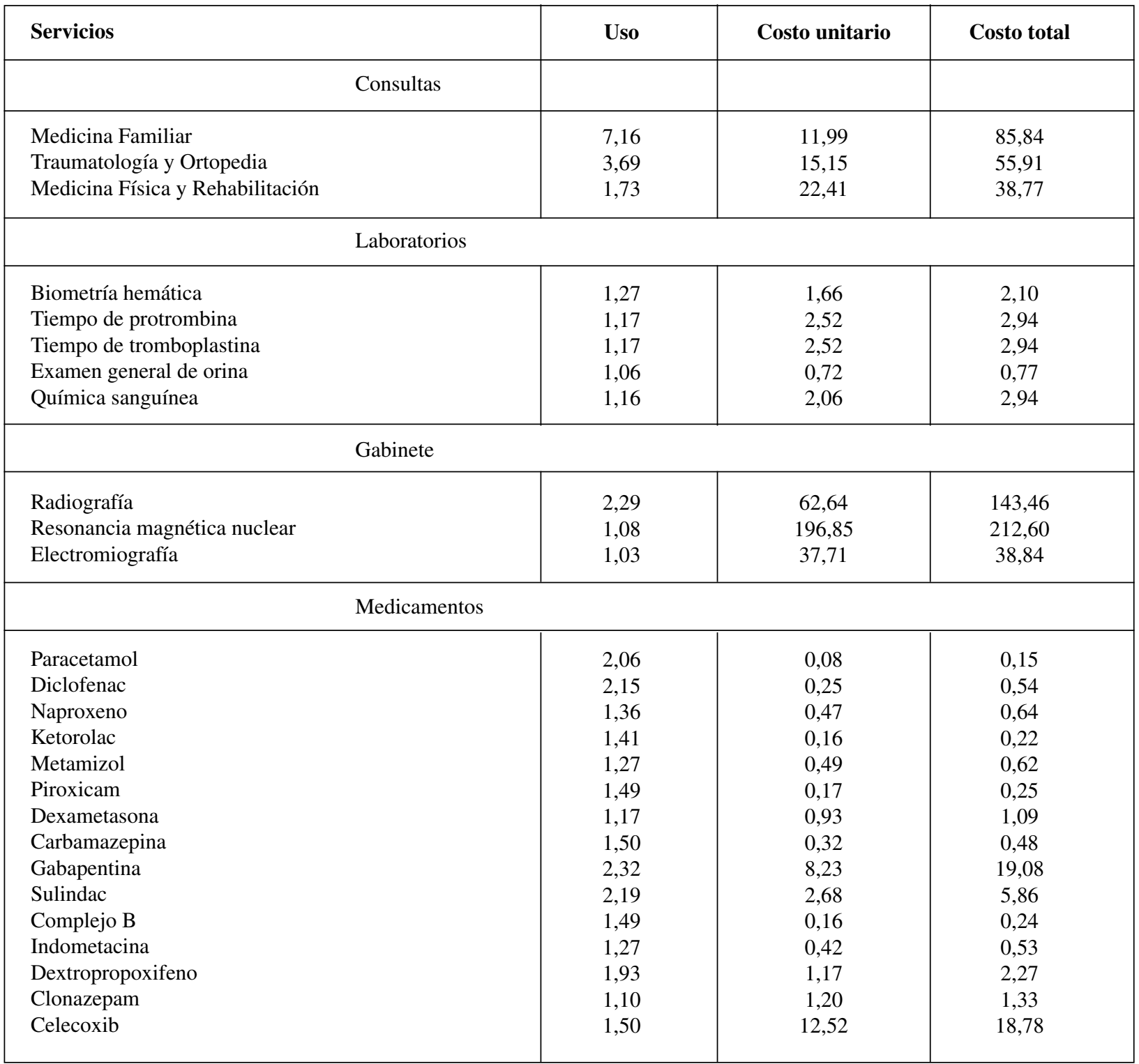

La prevalencia por tipo de lumbalgia se presenta similar a otras poblaciones, como lo demuestra en su estudio Arcila, en 2010, donde la lumbalgia por esfuerzo ocupa el primer lugar, seguida de la lumbociática y las hernias discales, y establece que las condiciones laborales y la falta de higiene postural son aspectos que deben analizarse, a fin de encontrar áreas de oportunidad que disminuyan su aparición, evolución y, por consiguiente, los costos institucionales. ${ }^{10}$

Covarruvias-Gómez, en 2010, menciona que el 13\% de la población (de 20 a 59 años) que acude a las Unidades de Medicina Familiar manifiesta este tipo de dolor, y comunica un mayor costo en el primer nivel, coincidiendo con el presente estudio, donde el mayor costo por servicio se ubica en Medicina Familiar en comparación con los
Servicios de Traumatología y Medicina Física; se explica en el hecho de que el médico familiar es quien toma las decisiones de envío a otros especialistas, además de que la evolución del paciente marca el número de consultas solicitadas. En 2007, Ocaña menciona que, en las sociedades occidentales, el número de consultas generadas en el primer nivel de atención fue de 2 millones. ${ }^{17,11}$

Dentro del proceso de atención, la indicación médica para el uso de la radiografía está establecida en la guía de práctica clínica y de acuerdo con el diagnóstico de mayor prevalencia encontrado en el presente estudio, no debe ser indicada a estos pacientes desde la primera consulta. $\mathrm{Al}$ no haber un seguimiento correcto de los lineamientos ya establecidos, el costo de los estudios de gabinete se incrementa y, por consiguiente, aumenta 
Tabla 3. Promedio de días y costo unitario promedio por tipo de incapacidad

\begin{tabular}{|c|c|c|c|c|c|c|c|c|c|}
\hline & \multicolumn{2}{|c|}{$\begin{array}{l}\text { Promedio } \\
\text { de días }\end{array}$} & \multicolumn{2}{|c|}{$\begin{array}{l}\text { Costo unitario } \\
\text { promedio }\end{array}$} & \multicolumn{3}{|c|}{$\begin{array}{c}\text { Costo } \\
\text { promedio }\end{array}$} & \multirow[t]{2}{*}{$\begin{array}{l}\text { Porcentaje } \\
\text { de pacientes }\end{array}$} & \multirow[t]{2}{*}{$\begin{array}{l}\text { Costo } \\
\text { total }\end{array}$} \\
\hline & $\begin{array}{l}\text { Riesgo } \\
\text { de } \\
\text { trabajo }\end{array}$ & $\begin{array}{l}\text { Enfer- } \\
\text { medad } \\
\text { general }\end{array}$ & $\begin{array}{c}\text { Riesgo } \\
\text { de } \\
\text { trabajo }\end{array}$ & $\begin{array}{l}\text { Enfer- } \\
\text { medad } \\
\text { general }\end{array}$ & $\begin{array}{l}\text { Riesgo } \\
\text { de } \\
\text { trabajo }\end{array}$ & $\begin{array}{l}\text { Enfer- } \\
\text { medad } \\
\text { general }\end{array}$ & $\begin{array}{c}\text { Riesgo/ } \\
\text { de trabajo } \\
\text { enfermedad } \\
\text { general }\end{array}$ & & \\
\hline $\begin{array}{l}\text { Riesgo } \\
\text { de trabajo }\end{array}$ & 71,93 & & 21,73 & & 1563,03 & & & 13,15 & 205,53 \\
\hline $\begin{array}{l}\text { Enfermedad } \\
\text { general }\end{array}$ & & 56,45 & & 25,92 & & 1019,99 & & 84,21 & 858,93 \\
\hline $\begin{array}{l}\text { Riesgo } \\
\text { de trabajo } \\
+ \text { enfermedad } \\
\text { general }\end{array}$ & 25,00 & 24,20 & 17,56 & 12,09 & 439 & 292,57 & 731,57 & 2,63 & 19,24 \\
\hline Costo total & & & & & & & & & 1083,70 \\
\hline
\end{tabular}

El costo se expresa en dólares estadounidenses.

el costo promedio total en la atención de pacientes con lumbalgia. ${ }^{7,12,13}$

El medicamento con el costo promedio más elevado fue la gabapentina, debido a la mayor frecuencia con la que se prescribe en el segundo nivel de atención, sobre todo si se combina con sulindac. Este tipo de medicamento es útil en lumbalgias con evolución crónica, principalmente si se presentan con dolor neuropático, es seguro y eficaz, como lo menciona Gilron en 2007, y mejora la calidad de vida. Un estudio realizado por Romano en 2012 reporta que la gabapentina combinada con otros analgésicos mejora la lumbalgia con dolor neuropático. ${ }^{14,15}$

La diferencia de costos en los diferentes tipos de incapacidades se presenta por el porcentaje que reciben del salario cotizado y el número de días otorgados por incapacidad, lo que se encuentra supeditado al diagnóstico y la evolución de la patología en el paciente. En México, el Consejero Médico de Incapacidades Médicas (MDA) determina el pago del salario en el caso de incapacidad

Tabla 4. Costo promedio del paciente con lumbalgia mecánica

\begin{tabular}{|l|c|}
\hline Servicios & Costo total \\
\hline Incapacidad & 1083,70 \\
Consultas & 180,52 \\
Laboratorio & 11,70 \\
Gabinete & 394,89 \\
Medicamentos & 52,09 \\
Costo total & 1722,9 \\
\hline
\end{tabular}

El costo se expresa en dólares estadounidenses. laboral, ya sea por riesgo de trabajo, donde se otorga el $100 \%$ del salario cotizado desde el primer día de ausencia laboral o si, es por enfermedad general, el 60\% del salario cotizado a partir del cuarto día. Si se acortara el tiempo para la práctica y entrega de estudios, y de tratamiento, y se llevara a cabo una evaluación más eficaz y completa del paciente, se podrían reducir los días de ITT.

$\mathrm{Al}$ estimar el costo promedio de la atención del paciente con lumbalgia, se considera elevado con un monto de 1744,08 USD, este resultado es mayor que el reportado en el estudio de Covarrubias-Gómez, en el que comunica que, en Europa, el 12\% de la población sufre incapacidad asociada a lumbalgia, con un costo por episodio de 101,66 USD o, en los EE.UU., donde la incidencia de incapacidades por lumbalgia es del $50 \%$ en la población económicamente activa, con un costo médico directo de 50.000 millones de dólares anuales. Al realizar una proyección a 2000 pacientes con dicha patología, resulta en un costo oneroso para el país, sobre todo, porque la vida laboralmente productiva se detiene, situación que se agrava cuando el paciente está incapacitado de manera prolongada. ${ }^{16}$

\section{Conclusiones}

Se puede decir que el costo institucional del paciente con incapacidad temporal para el trabajo, con lumbalgia mecánica resulta en miles de dólares y, proyectado a una población de pacientes, el costo se incrementa a millones. 
Tabla 5. Proyección del costo promedio total de pacientes

\begin{tabular}{|c|c|c|c|c|c|c|}
\hline \multirow{2}{*}{ Servicios } & \multicolumn{6}{|c|}{ Cantidad de pacientes } \\
\hline & 1 & 50 & 100 & $\mathbf{5 0 0}$ & 1000 & 2000 \\
\hline \multicolumn{7}{|c|}{ Incapacidad } \\
\hline Costo promedio & 1083,70 & $54.185,00$ & $108.370,00$ & $541.850,00$ & $1.083 .700,00$ & $2.167 .400,00$ \\
\hline \multicolumn{7}{|c|}{ Consultas } \\
\hline Medicina Familiar & 85,84 & 4291,89 & 8583,78 & $42.918,88$ & $85.837,76$ & $171.675,53$ \\
\hline Traumatología y Ortopedia & 55,91 & 2795,34 & 5590,69 & $27.953,45$ & $55.906,90$ & $111.813,81$ \\
\hline Medicina Física y Rehabilitación & 38,77 & 1938,63 & 3877,26 & $19.386,32$ & $38.772,63$ & $77.545,26$ \\
\hline \multicolumn{7}{|c|}{ Laboratorios } \\
\hline Biometría hemática & 2,10 & 105,18 & 210,35 & 1051,76 & 2103,52 & 4207,04 \\
\hline Tiempo de protrombina & 2,94 & 147,17 & 294,33 & 1471,67 & 2943,34 & 5886,68 \\
\hline Tiempo de tromboplastina & 2,94 & 147,17 & 294,33 & 1471,67 & 2943,34 & 5886,68 \\
\hline Examen general de orina & 0,77 & 38,26 & 76,52 & 382,59 & 765,17 & 1530,35 \\
\hline Química sanguínea & 2,94 & 147,17 & 294,33 & 1471,67 & 2943,34 & 5886,68 \\
\hline \multicolumn{7}{|c|}{ Gabinete } \\
\hline Radiografías & 143,46 & 7172,76 & $14.345,51$ & $71.727,56$ & $143.455,11$ & $286.910,23$ \\
\hline Resonancia magnética nuclear & 212,60 & $10.629,82$ & $21.259,64$ & $106.298,21$ & $212.596,43$ & $425.192,86$ \\
\hline Electromiografía & 38,84 & 1942,12 & 3884,24 & $19.421,19$ & $38.842,38$ & $77.684,76$ \\
\hline \multicolumn{7}{|c|}{ Medicamentos } \\
\hline Paracetamol & 0,15 & 7,71 & 15,41 & 77,07 & 154,13 & 308,26 \\
\hline Diclofenac & 0,54 & 27,15 & 54,30 & 271,49 & 542,98 & 1085,95 \\
\hline Naproxeno & 0,64 & 31,92 & 63,84 & 319,21 & 638,43 & 1276,86 \\
\hline Ketorolac & 0,22 & 11,03 & 22,06 & 110,32 & 220,63 & 441,27 \\
\hline Metamizol & 0,62 & 30,79 & 61,57 & 307,87 & 615,74 & 1231,48 \\
\hline Piroxicam & 0,25 & 12,67 & 25,35 & 126,75 & 253,49 & 506,99 \\
\hline Dexametasona & 1,09 & 54,45 & 108,91 & 544,54 & 1089,08 & 2178,17 \\
\hline Carbamazepina & 0,48 & 24,18 & 48,35 & 241,76 & 483,52 & 967,03 \\
\hline Gabapentina & 19,08 & 954,20 & 1908,40 & 9541,99 & $19.083,98$ & $38.167,96$ \\
\hline Sulindac & 5,86 & 292,96 & 585,93 & 2929,65 & 5859,30 & $11.718,59$ \\
\hline Complejo B & 0,24 & 12,21 & 24,41 & 122,05 & 244,10 & 488,21 \\
\hline Indometacina & 0,53 & 26,72 & 53,44 & 267,19 & 534,37 & 1068,74 \\
\hline Dextropropoxifeno & 2,27 & 113,25 & 226,50 & 1132,51 & 2265,01 & 4530,02 \\
\hline Clonazepam & 1,33 & 66,27 & 132,54 & 662,68 & 1325,36 & 2650,73 \\
\hline Celecoxib & 18,78 & 938,86 & 1877,73 & 9388,64 & $18.777,29$ & $37.554,57$ \\
\hline Costo total & 1722,9 & $86.144,88$ & $172.289,72$ & $861.448,69$ & $1.722 .897,33$ & $3.445 .794,71$ \\
\hline
\end{tabular}

\section{Bibliografía}

1. Covarrubias-Gómez A. Lumbalgia: Un problema de salud pública. Revista Mexicana de Anestesiología 2010;33:106-9.

2. Pérez J. Contribución al estudio de la lumbalgia inespecífica. Rev Cubana Ortop Traumatol 2006;20(2):1-25.

3. Duque I, Zuluaga D, Pinilla A. Prevalencia de lumbalgia y factores de riesgo en enfermeros y auxiliares de la ciudad de Manizales. Hacia la Promoción de la Salud 2011;16:27-38.

4. Araña-Suárez SM. Trastornos musculoesqueléticos, psicopatología y dolor. Programa Nacional de FIPROS 2009;(38). 
5. Noriega-Elío M, Barrón A, Sierra O, Méndez I, Pulido M, Cruz C. La polémica sobre las lumbalgias y su relación con el trabajo: estudio retrospectivo en trabajadores con invalidez. Cad Saúde Pública 2005;21(3):887-97.

6. Instituto Mexicano del Seguro Social. Guía de práctica clínica diagnóstica, tratamiento y prevención de lumbalgia aguda y crónica en el primer nivel de atención 2008.

7. Ocaña U. Lumbalgia ocupacional y discapacidad laboral. Revista de Fisioterapia 2007;6(2):17-26.

8. Saldívar A, Cruz D, Serviere L, Vázquez F, Joffre V. Lumbalgia en trabajadores. Epidemiología. Rev Med IMSS 2003;41(3):203-9.

9. Hallne D, Hesembring M. Classification of psychosocial risk factors (yellow flags) for the development of chronic low back and leg pain using artificial neural network. Neuroscience Letters 2004;361(1-3):151-4.

10. Arcila-Herrera H. Patologías del sistema musculoesquelético. Rev Med CMA 2010;3:4-9.

11. Sánchez-Hernández M, Ibarra-Ramírez C, Vilchis-Aguado M, Montesano-Delfín J, Guízar-Mendoza J, Loya-García B. Impacto del servicio de rehabilitación en el primer nivel de atención. Rev Med Inst Mex Seguro Soc 2008;(4):391-6.

12. Airaksinen O, Brox JI, Cedraschi C, Hildebrandt J, Klaber-Moffett J, Kovacs F, et al. European guidelines for the management of chronic nonspecific low back pain. Eur Spine J 2006;15(Suppl 2):S192-300.

13. Boleaga B. Lumbalgia. Anales de Radiología México 2005;(2):89-97.

14. Romanó C, Romanó D, Lacerenza M. Antineuropathic and antinociceptive drugs combination in patients with chronic low back pain. A systematic review 2012; (2012), Article ID 154781, 8 pages. doi:10.1155/2012/154781. [Consulta: 20 de marzo de 2013].

15. Gilron I. Gabapentin and pregabalin for chronic neuropathic and early postsurgical pain: current evidence and future directions. Curr Opin Anaesthesiol 2007;20(5):456-72.

16. Young R, Benold T, Whitham J, Burge S. Factors influencing work interference in patients with chronic low back pain: a residency research network of texas. (RRNeT) Study 2011;(24):503-10. [Consulta: 10 de septiembre de 2012] Disponible en: http:// www.jabfm.org/content/24/5/503.full.pdf. 